ONLINE MUTATION REPORT

\title{
Six families with van der Woude and/or popliteal pterygium syndrome: all with a mutation in the IRF6 gene
} M Ghassibé, N Revencu, B Bayet, Y Gillerot, R Vanwijck, C Verellen-Dumoulin, and M Vikkula

J Med Genet 2004;41:e15 (http://www.jmedgenet.com/cgi/content/full/41/2/e15). doi: 10.1136/jmg.2003.009274

$\mathrm{V}$ an der Woude syndrome (VWS, OMIM \#119300) is a dominantly inherited developmental disorder characterised by pits and/or sinuses of the lower lip, cleft lip with or without cleft palate $(\mathrm{CL} / \mathrm{P})$, isolated cleft palate $(\mathrm{CP})$, bifid uvula (BU), and hypodontia $(\mathrm{H}) \cdot{ }^{1-3}$ Cleft lip deformity is established during the first 6 weeks of life due to failure of fusion of maxillary and medial nasal processes or to incomplete mesodermal ingrowth into the processes. Palatal clefts result from failure of fusion of the palatal shelves that normally change from a vertical to horizontal position and fuse during the sixth to ninth weeks of gestation.

The severity of VWS varies widely, even within families. About $25 \%$ of individuals with the syndrome have minimal findings, such as absent teeth or trivial indentations in the lower lips. Clefting of the lip or palate is present in $\sim 50 \%$ of cases. Lip pits and/or sinuses are the cardinal features of this syndrome, present in $70-80 \%$ of VWS patients. ${ }^{2}$ They are often associated with accessory salivary glands that empty into the pits, sometimes leading to embarrassing visible discharge. ${ }^{45}$ Another syndrome closely mimicking VWS is popliteal pterygium syndrome (PPS, OMIM \#119500). It combines VWS signs and symptoms with popliteal and oral webs, unusual nails, syndactyly, ankyloblepharon, and genital anomalies ${ }^{6}$ (fig 1).

VWS is the most common cleft syndrome, affecting 1 in $35000-100000$ people $(\sim 1 / 70000) . .^{7}$ About $1-2 \%$ of patients with cleft lip or palate have VWS. The VWS pedigrees suggest an autosomal dominant inheritance pattern, yet, in 30-50\% of cases, mutation may rise de novo. ${ }^{8}$ Penetrance is estimated to be about $75 \%$, but is actually closer to $100 \%$ when supposedly unaffected carriers are closely examined for minor expressions of the syndrome. ${ }^{24}$ PPS is a rarer malformation, with an incidence of $1 / 300000 .^{9}$

Most reported cases of VWS have been linked to chromosome 1q32-q41 (VWS1), ${ }^{10-12}$ but a second VWS locus (VWS2) has been mapped to $1 \mathrm{p} 34 .{ }^{13}$ Recently, the interferon regulatory factor-6 (IRF6) gene, localised to the VWS1 locus on chromosome band 1q32.2, was shown to harbour mutations in patients with Van der Woude and/or popliteal pterygium syndrome. ${ }^{14}$

IRF6 belongs to a family of nine transcription factors that share a highly conserved helix turn helix DNA binding domain and a less conserved protein binding domain called SMIR. ${ }^{15}{ }^{16}$ In the only report so far on mutations in the IRF6 gene in VWS and PPS patients, not all families harboured an alteration in IRF6. ${ }^{14}$ Thus, analysis of novel families, with or without PPS, is interesting in order to determine the prevalence of IRF6 as a disease causative gene and to identify additional mutations responsible for VWS with or without PPS. In this study, we screened six VWS and PPS families followed at the Centre Labiopalatin for mutations. A novel mutation was identified in IRF6 for all the cases.

\section{Key points}

- Van der Woude syndrome is a dominantly inherited developmental disorder characterised by pits and/or sinuses of the lower lip, cleft lip with or without cleft palate, isolated cleft palate, bifid uvula, and hypodontia.

- Its severity varies widely, even within families, with about a quarter having minimal findings, and about half having cleft lip or palate.

- Lip pits or sinuses are the cardinal features.

\section{MATERIALS AND METHODS}

Family assessment

Patients diagnosed as VWS were recalled from the registries of the Cleft Lip and Palate Centre and the Centre for Human Genetics. All the families had members with clefts of the lip and/or palate, lip pits, or bifid uvula, and were thus considered VWS patients. In family VWS5, individuals IV:1 and IV:3 had, in addition to VWS features, oral synechiae and syndactyly, which are signs of PPS. The clinical data for each individual are shown in figure 2. Each participant gave informed consent prior to his/her participation in the study, as approved by the ethics committee of the medical faculty at the Université catholique de Louvain. The status of the unaffected individuals has been ascertained in order to identify all the microforms of VWS, especially small lip sinuses and hypodontia.

For genomic DNA extraction, venous blood samples were drawn for all participants other than VWS11-II:1, for whom a buccal cell sample was obtained. DNA was extracted by isopropanol precipitation or, from the buffy coats, using QIAamp DNA Blood Mini Kit (Qiagen, Westburg, Leusden, The Netherlands). A second blood sample was obtained from some individuals for lymphocytic transformation with Epstein-Barr virus.

\section{Genotyping}

Genotyping of nine individuals of the VWS2 family was performed as described elsewhere. ${ }^{17}$ Linkage was tested using four polymorphic microsatellite markers: D1S245, D1S491, D1S205, and D1S414, on the basis of their genetic location given by the Genome DataBase (http://gdbwww.gdb.org/). These markers flank the VWS I critical region and are spaced

Abbreviations: PPS, popliteal pterygium syndrome; VWS, Van der Woude syndrome 
at $\sim 1$ cM intervals. The radioactively labelled PCR products for each marker were resolved on polyacrylamide gels. In order to test linkage between the disease and the IRF6 gene, two point analyses of disease status versus marker alleles were carried out manually, under the hypothesis of dominant inheritance. All individuals with CL/P, pits or sinuses, bifid uvula, hypodontia, or a combination of these symptoms were considered as affected. On the basis of the four markers, haplotypes were manually generated across the VWS region.

\section{Mutational screening}

The genomic sequences containing the IRF6 gene were identified by performing a Blast search with the IRF6 mRNA sequence (NM_006147) on the human genome sequence at the NCBI Blast server (http://www.genome.ucsc. edu/cgi-bin/). The results were confirmed by the genomic sequence retrieved. Eighteen intronic primers (sequences available on request) were synthesised from the genomic sequence to amplify the coding exons 2-9 of IRF6, including the intron-exon boundaries. The gene consists of 10 exons, the first and the last being non-coding. Genomic IRF6 DNA was screened by radioactive SSCP and heteroduplex analysis for individuals VWS1-II:2; VWS1-III: 1; WS2-IV:2; VWS2-V:1; VWS5-III:6; VWS5-III:1; VWS6-I:1; VWS6-II:2; VWS7-III:4; VWS7-IV:3; VWS11-II:1; VWS11-III:2 (fig 2), as described elsewhere. $^{18}$ Amplified fragments were also loaded onto denaturing $5 \%$ acrylamide sequencing gels to identify potential insertions or deletions. Fragments with abnormal migration were reamplified, purified, and sequenced on a CEQ2000 capillary sequencer (Beckman Coulter, Analis, Namur, Belgium), using the intronic primers mentioned above, with unaffected individuals from the same family as controls.

\section{Co-segregation of mutations}

Co-segregation of the substitutional mutations with the phenotype was studied by restriction fragment length assay. Numbering of nucleotides is based on the cDNA sequence (NM 0061147), with the adenosine of the ATG start codon marked as +1 . The $65 \mathrm{~T} \rightarrow \mathrm{C}$ substitution in family VWS5 created a new NlaIV restriction enzyme cutting site, whereas the $755 \mathrm{~T} \rightarrow \mathrm{C}$ substitution in family VWSI abolished the MboII restriction enzyme cutting site, and the $47 \mathrm{C} \rightarrow \mathrm{T}$ substitution in family VWS6 abolished the HaeIII restriction enzyme cutting site. As the 191C $\rightarrow$ T substitution in family VWS2 and the $775 \mathrm{C} \rightarrow \mathrm{T}$ mutation in family VWS11 did not change any restriction site, allele specific PCR was used. For family VWS2, we synthetised wild type and mutant primers for the substitution site (5'-GCCTGGGCTGTAGAGAC/T-3'), and used them in combination with a reverse primer (3'ACCTTCTCCCCAGCACCT-5'). For family VWS11, the wildtype and mutant primers ( 3 '-TCCTGGTCAGGCAT GGG/A-5') were used in combination with a forward primer of exon 7 (5'-TCTCACTGTCATCTCT AACCTTGC-3'). The annealing temperature was $57.1{ }^{\circ} \mathrm{C}$ for family VWS2 and $62{ }^{\circ} \mathrm{C}$ for family VWSil. A reverse primer (3'-TTCTCATT GGTAATATGCTCAGGA-5') for family VWS11 was added to the reaction to serve as an internal positive control and as a competitor to improve the specificity of the reaction. For family VWS7, the co-segregation of the mutation was confirmed by SSCP. Fifty unrelated healthy controls were screened for all the mutations using the corresponding methods.
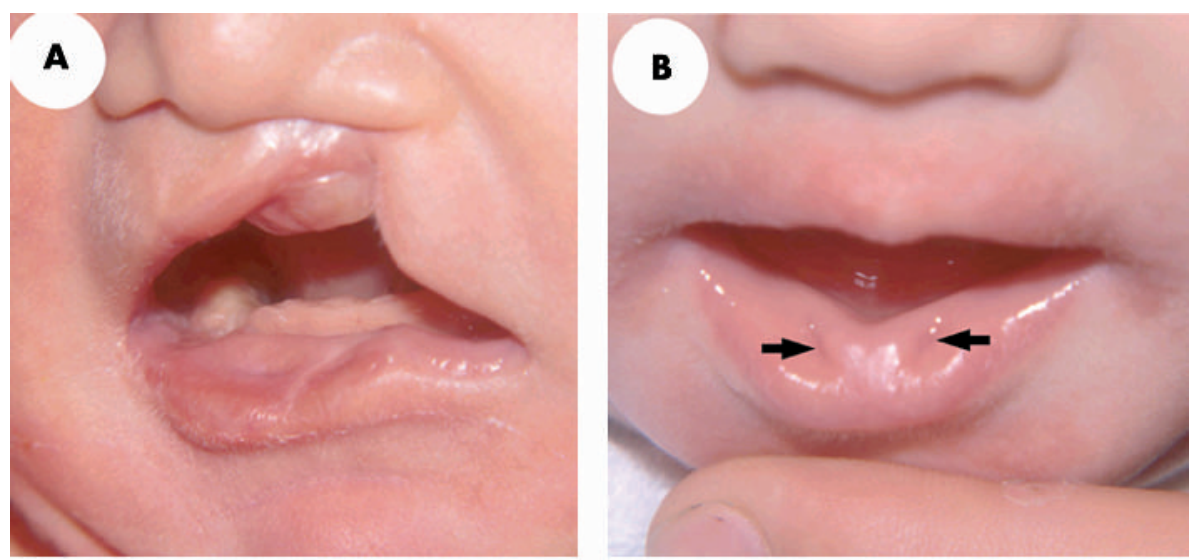

Figure 1 The major features of VWS and PPS. (A) Unilateral cleft lip in VWS5-IV:3; (B) lip pits in VWS5-IV: 1; (C) syndactyly of fingers in WWS5-IV:1; (D) buccal synechiae in WWS5-IV:3 The position of the lip pits is shown by the arrow and the arrowhead indicates the buccal synechiae.
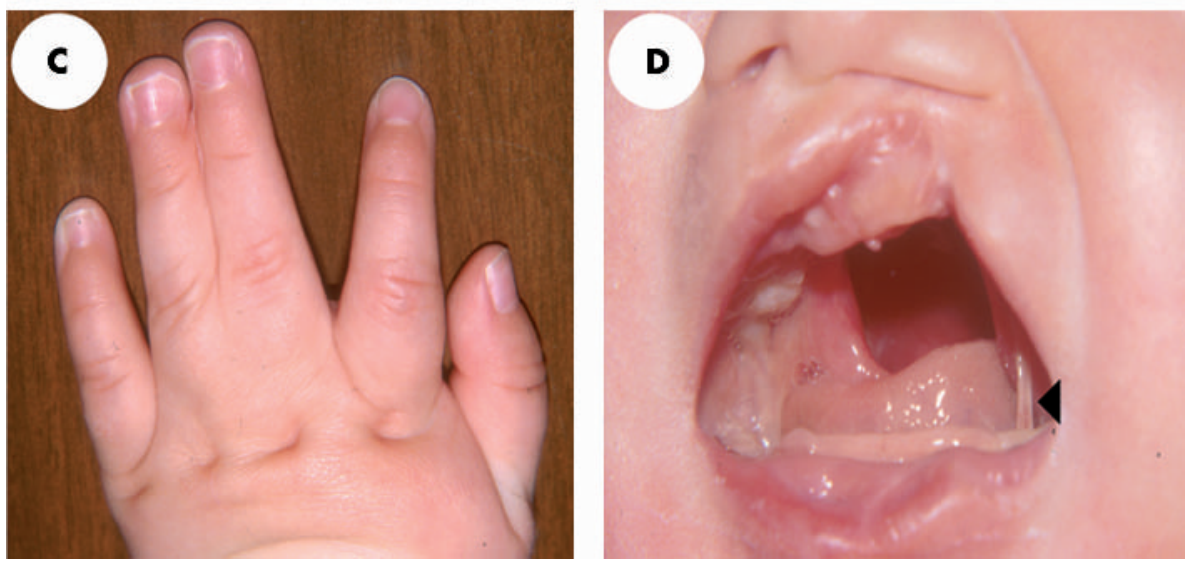

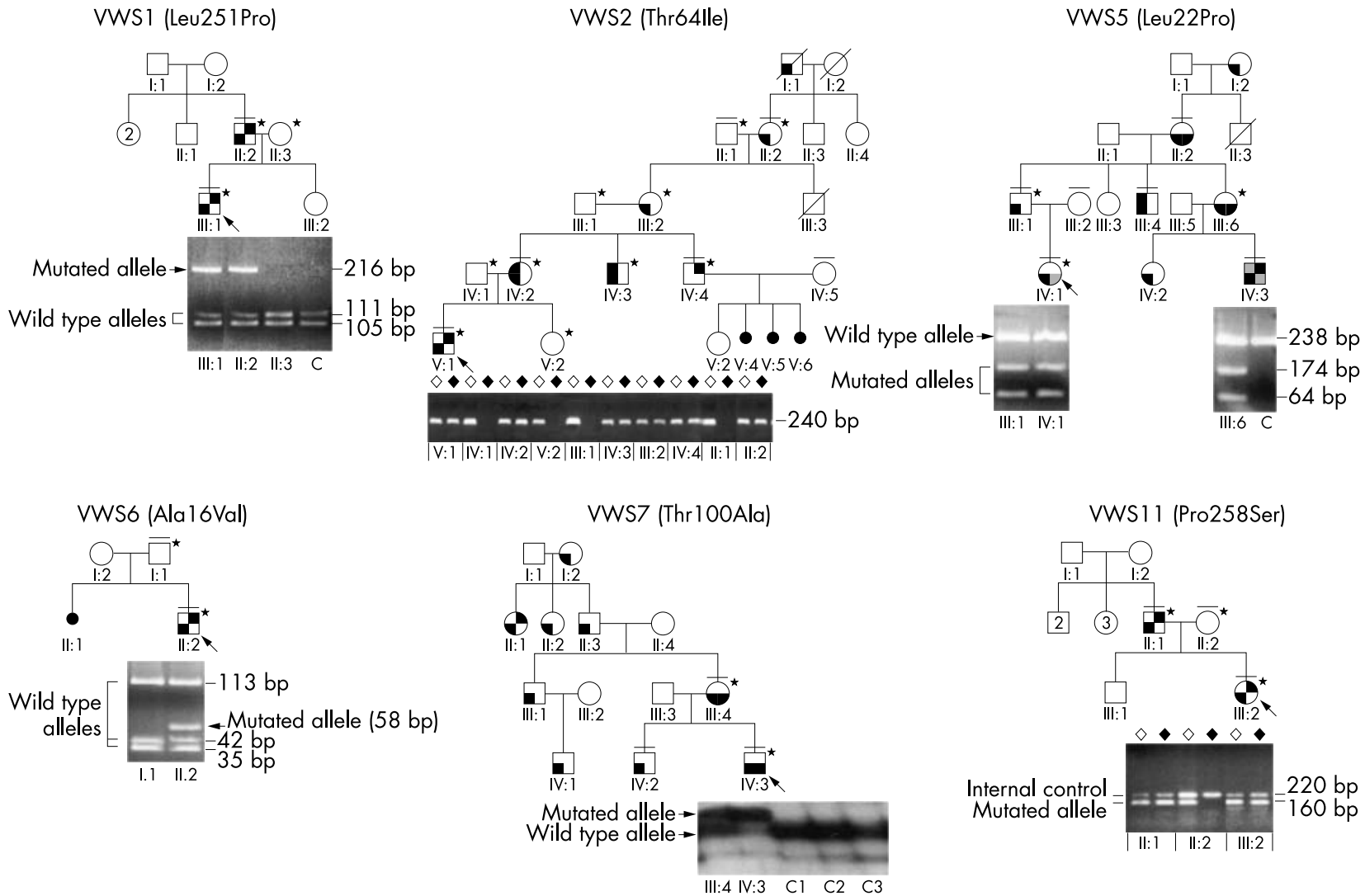

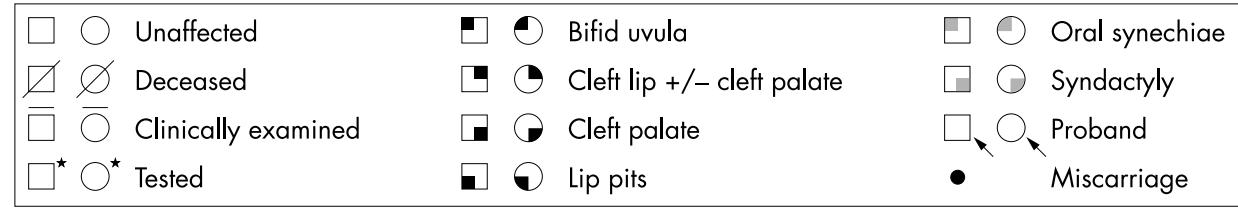

Figure 2 Pedigrees of six families with van der Woude and popliteal pterygium syndrome. A photograph of an agarose gel underneath each pedigree shows the segregation of the allele harbouring the substitution. VWS1, VWS5, VWS6: restriction enzyme digestion; VWS2, VWS1 1: allele specific PCR. VWS7: single strand conformational polymorphism (SSCP) analysis. $\diamond$, wild type allele; $\bullet$, mutant allele.

\section{RESULTS}

\section{Clinical characterisation and linkage analysis to the} 1q32-q41 VWS locus

Clinical examination of six recalled patients with VWS (VWS1-III:1; VWS2-V:1; VWS3-IV:1; VWS4-II:2; VWS5-IV:3 and VWS6-III:2) revealed that five had at least one other affected family member, and for patient VWS6-III:2, there was no known family history of the disease (fig 2). Lip pits were present in $\sim 90 \%$ of affected individuals (28/29), confirming that it is a major VWS symptom. The number of affected males and females in the six families was 16 and 13, respectively, and $72 \%(23 / 32)$ of children of an affected parent had the disorder. Examination of the pedigrees revealed that the condition did not skip any generation. Thus, an autosomal dominant pattern of inheritance with very high penetrance and absence of sex bias was observed. In family VWS5, IV:1 and IV:3 were affected with VWS and syndactyly of fingers, and IV:3 had oral synechiae.

Linkage analysis in the VWS1 locus on 1q32-q41, performed for the nine members of the VWS2 family (three unaffected individuals and six individuals with VWS), demonstrated co-segregation of marker alleles and a defined haplotype with the phenotype (data not shown), considering as affected each individual who had one or more of the VWS features.

\section{Identified mutations}

Screening the IRF6 gene revealed six heterozygous substitutions detected in the six families analysed (fig 3). All were missense mutations affecting either the DNA binding domain or the protein binding domain of the transcription factor IRF6 (fig 4). The missense mutations detected in families VWS2, VWS5, VWS6, and VWS7 were Thr64Ile $(191 \mathrm{~T} \rightarrow \mathrm{C})$, Leu22Pro $(65 \mathrm{~T} \rightarrow \mathrm{C})$, Alal6Val $(47 \mathrm{C} \rightarrow \mathrm{T})$, and Thrl00Ala $(298 \mathrm{~A} \rightarrow \mathrm{G})$, respectively. They all affected the DNA binding domain of IRF6 (fig 4). The substitutions in families VWS1 and VWS11, Leu251Pro $(755 \mathrm{~T} \rightarrow \mathrm{C})$, and Pro258Ser $(775 \mathrm{C} \rightarrow \mathrm{T})$, respectively, affected the protein binding domain of IRF6. Co-segregation of the mutant alleles with the VWS phenotype was confirmed in all five familial cases (fig 2). There were no unaffected carriers, probably because all family members were examined for minor expressions of the syndrome. None of the mutations was observed in the 100 control alleles screened, and no other nucleotide changes were identified.

\section{DISCUSSION}

In this study, we describe six new families with autosomal dominantly inherited VWS caused by mutations in the IRF6 gene localised at 1q32.2. In family VWS5, patient IV:1 had finger syndactyly, and IV:3 had toe syndactyly and oral 


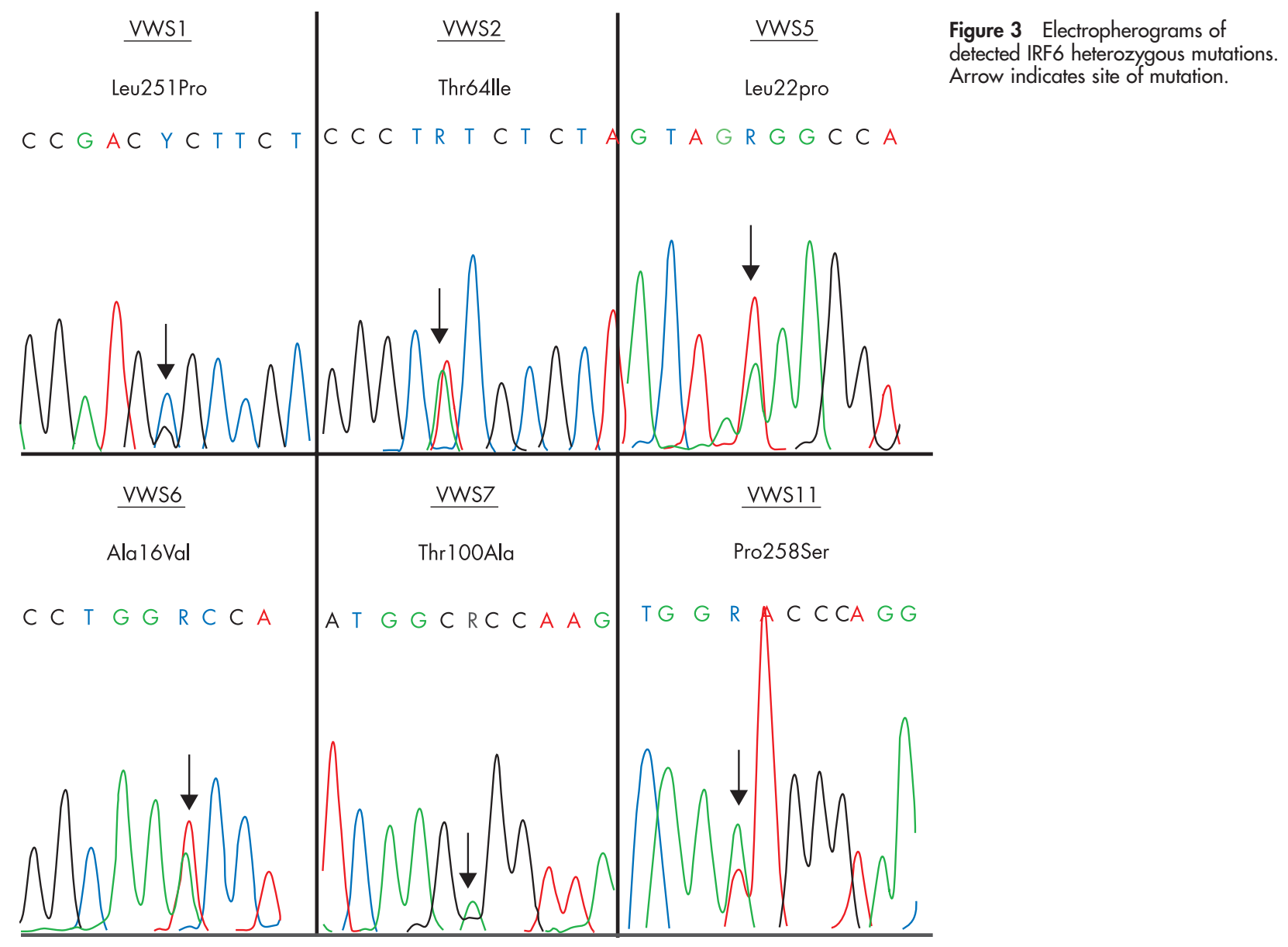

synechiae, in addition to signs of VWS. As these two features are considered the major PPS symptoms, these two individuals were taken as PPS patients. The other affected members of this family presented the VWS signs and were thus classified as VWS patients (fig 2). Another affected individual in this family, III:4, had a canine tooth in the palate. These data confirm the results of the previous study ${ }^{14}$ that mutations in the IRF6 gene are causative for VWS and PPS. It shows also that a single mutation in IRF6 can be responsible for both syndromes. Both isolated cleft palate and clefts of the lip with or without cleft palate were noted within the same family, confirming that VWS, like the ectodermal dysplasia clefting syndromes, is one of the rare syndromes causing both cleft lip and palate, which, when isolated, are thought to be genetically and embryologically distinct. ${ }^{19} 20$

In the present study, all detected mutations were novel missense mutations affecting the well conserved DNA binding domain, or the less conserved protein binding domain that is needed for homo- and hetero-dimerisation of IRF6. ${ }^{21}{ }^{22}$ As two of the three residues mutated in the DNA binding domain are involved in interaction with DNA, the substitutions could cause VWS by loss of function. This may be caused by the mutations altering the three dimensional structure of IRF6, making it unable to bind DNA. Similarly, the protein binding domain substitutions could alter dimerisation of the transcription factor.

Loss of function is supported by the detection of a number of large deletions encompassing the VWSI region in some patients in an earlier study. ${ }^{10}$ Haploinsufficiency was also underscored by the identification of several protein truncating mutations in the IRF6 gene that were more common in VWS than in PPS. ${ }^{14}$

The single PPS family studied (VWS5), harboured a Leu22Pro mutation. The Leu22 residue in IRF6 is comparable to Gln20 in IRF1, which belongs to helix-1 of the DNA binding domain, but does not interact with DNA. ${ }^{23}$ In a previous study, the majority of PPS missense mutations was located in the DNA binding domain and was suggested to

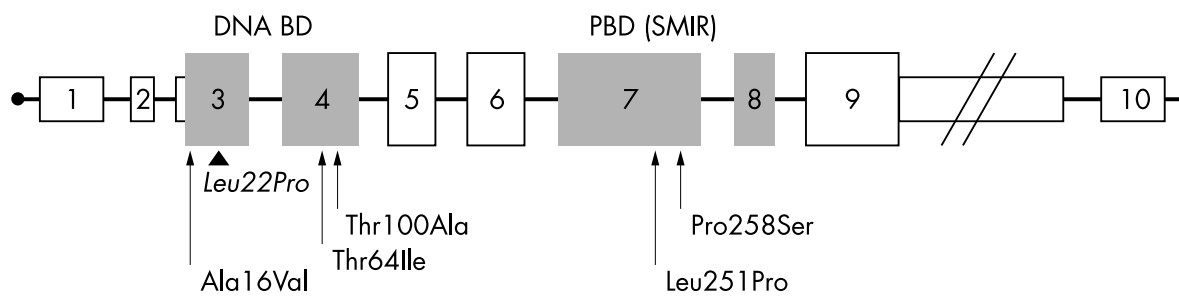

Figure 4 Structure of the IRF6 gene. Exons (rectangles) are drawn to scale, except for exon 9, which is longer than shown. Line connecting exons represents introns. Untranslated regions are in grey. Exons $3-4$ code for the DNA binding domain and exons 7-8 code for the protein binding domain (both in grey). The arrowhead indicates the relative position of the identified PPS mutation, and the arrows the relative positions of the other substitutions. 
lead to an inactive form of the transcription complex in a dominant negative manner. ${ }^{14}$ Individuals in our VWS5 family had either VWS or PPS caused by the same mutation. Therefore, it seems rather unlikely that the missense mutations in the DNA binding domain associated with VWS or PPS affect IRF6 function differently. ${ }^{14}$

The sequence variant Val274Ile, which is reported to be present in 3\% of individuals of European descent and 22\% of Asian population, ${ }^{14}$ was not detected in any of the 22 white individuals analysed. Thus, it does not seem to be a phenotypic modifier in this subgroup. However, as wide variation is observed in the phenotypic effects of the detected mutations in the IRF6 gene, it is likely that other unknown stochastic factors or modifier genes influencing IRF6 function exist.

In the present study, 5/6 IRF6 mutations were hereditary, detected in familial VWS/PPS. None was a confirmed de novo mutation. However, in three families (VWS1, VWS6, and VWS11), we cannot exclude this possibility, owing to limited familial history and DNA samples. A mutation was detected in all the affected individuals of the families. This is an expected result for a PPS family, as a mutation in the IRF6 gene was previously detected in $\sim 86.6 \%$ of PPS families ( $13 /$ 15). ${ }^{14}$ In contrast, we did not expect to reveal mutations in all the VWS patients, as only 43\% (46/107) of VWS families had an IRF6 mutation in the previous study [154]. This may be due to our smaller sample size, or to the fact that the families were phenotypically more homogeneous.

VWS, one of the most common cleft syndromes in the world, is the best example of a single gene disorder that causes cleft lip and palate, and which can mimic a "nonsyndromic" form. For this reason, the identification of the IRF6 gene as the cause of VWS and PPS can help in the complex puzzle of cleft lip and palate, but how does IRF6 cause clefts of palate and lips, and other variable craniofacial phenotypes? Very little is known about the function of IRF6. It is a transcription factor with two conserved domains, with the structure of an interferon regulatory factor gene, or IRF. It is not known which target genes it activates and when. They could be extracellular matrix genes, such as collagens that have been shown to be mutated in other syndromic forms of clefts, or genes acting in the TGF $\beta$ pathway, for example, which has been shown to be important for palate formation. ${ }^{24-26}$ It could also act as a modifier, which by itself does not cause a cleft, but which affects the severity of the cleft, due to alterations in other proteins. Modifying effects could be due to expression level differences and thus, screening of, for example, the IRF6 promoter may unravel cleft lip/palate associated variants.

\section{ACKNOWLEDGEMENTS}

We are grateful to all the families for their participation in the study. The studies were supported by the Fonds Spéciaux de RechercheUniversité catholique de Louvain, the Fonds National de la Recherche Scientifique (FNRS) (to M Vikkula, a "chercheur qualifié du FNRS"), and by La Communauté Française de Belgique. M Ghassibé was supported by a fellowship from the John B Mulliken foundation and from Fonds pour la Formation à la Recherche dans l'Industrie et dans l'Agriculture). The authors thank Ms A Gutierrez for her expert technical assistance, and Ms L Niculescu for secretarial help.

\section{Authors' affiliations}

M Ghassibé, M Vikkula, Laboratory of Human Molecular Genetics, Christian de Duve Institute of Cellular Pathology and Universite catholique de Louvain, Avenue Hippocrate 74+4, Brussels, Belgium N Revencu, Y Gillerot, C Verellen-Dumoulin, Centre for Human Genetics, Cliniques universitaires St Luc and Université catholique de Louvain, Brussels, Belgium
B Bayet, R Vanwijck, Centre Labiopalatin, Division of Plastic Surgery, Cliniques universitaires St Luc, Brussels, Belgium

Correspondence to: Professor M Vikkula, Laboratory of Human Molecular Genetics, Institute of Cellular Pathology and Universite catholique de Louvain, Avenue Hippocrate 74+4, Brussels B-1200, Belgium; vikkula@bchm.ucl.ac.be

\section{REFERENCES}

1 Van der Woude A. Fistula labii inferioris congenita and its association with cleft lip and palate. Am J Hum Genet 1954;6:244-56.

2 Janku P, Robinow M, Kelly T. The van der Woude syndrome in a large kindred: variability, penetrance, genetic risks. Am J Med Genet 1980;5:117-23.

3 Lacombe D, Pedespan JM, Fontan D. Phenotypic variability in van der Woude syndrome. Genet Couns 1995;6:221-6.

4 Menko FH, Koedijk PH, Baart JA. van der Woude syndrome-recognition of lesser expressions: case report. Cleft Palate J 1988;25:318-21.

5 Ranta R, Rintala AE. Correlations between microforms of the van der Woude syndrome and cleft palate. Cleft Palate J 1983;20:158-62.

6 Bixler D, Poland C, Nace WE. Phenotypic variation in the popliteal pterygium syndrome. Clin Genet 1973;4:220-8

7 Gorlin RJ, Cohen MM Jr, Hennekam RCM. Orofacial clefting syndromes: common syndromes. In: Motulsky AG, Bobrow M, Harper PS, Scriver C, eds. Syndromes of the head and neck, 4th edn. New York: Oxford University Press, 2001.

8 Conners PG. van der Woude syndrome. eMedicine Journal 2002;4

Froster-Iskenius UG. Popliteal pterygium syndrome. J Med Genet 1990;27:320-6.

10 Bocian M, Walker AP. Lip pits and deletion 1q32-q41. Am J Med Genet 1987;26:437-43.

11 Murray JC, Nishimura DY, Buetow KH, Ardinger HH, Spence MA, Sparkes RS Falk RE, Falk PM, Gardner RJM, Harkness EM, Glinski LP, Pauli RM, Nakamura Y, Green PP, Schinzel A. Linkage of an autosomal dominant clefting syndrome (van der Woude) to loci on chromosome 1q. Am J Hum Genet 1990:46:486-91.

12 Schutte BC, Bjork BC, Coppage KB, Malik MI, Gregory SG, Scott DJ, Brentzell LM, Watanabe Y, Dixon MJ, Murray JC. A preliminary gene map for the Van der Woude syndrome critical region derived from $900 \mathrm{~kb}$ of genomic sequence at 1q32-q41. Genome Res 2000;10:81-94.

13 Koillinen H, Wong FK, Rautio J, Ollikainen V, Karsten A, Larson O, Teh BT, Huggare J, Lahermo P, Larsson C, Kere J. Mapping of the second locus for the van der Woude syndrome to chromosome 1p34. Eur J Hum Genet 2001;9:747-52.

14 Kondo S, Schutte BC, Richardson RJ, Biork BC, Knight AS, Watanabe Y Howard E, Ferreira de Lima RLL, Daack-Hirsch S, Sander A, McDonaldMcGinn DM, Zackai EH, Lammer EJ, Aylsworth AS, Ardinger HH, Lidral AC, Pober BR, Moreno L, Arcos-Burgos M, Valencia C, Houdayer C, Bahuau M, Moretti-Ferreira D, Richieri-Costa A, Dixon MJ, Murray JC. Mutations in IRF6 cause Van der Woude and popliteal pterygium syndromes. Nature Genet 2002;32:285-9.

15 Taniguchi T, Ogasawara K, Takaoka A, Tanaka N. IRF family of transcription factors as regulators of host defense. Annu Rev Immunol 2001;19:623-55.

16 Eroshkin A, Mushegian A. Conserved transactivation domain shared by interferon regulatory factors and Smad morphogens. J Mol Med 1999;77:403-5.

17 Boon LM, Mulliken JB, Vikkula M, Watkins H, Seidman J. Olsen BR, Warman ML. Assignment of a locus for dominantly inherited venous malformations to chromosome 9p. Hum Mol Genet 1994;3:1583-87.

18 Boon LM, Brouillard P, Irrthum A, Karttunen L, Warman ML, Rudolph R, Mulliken JB, Olsen BR, Vikkula M. A gene for inherited cutaneous venous anomalies ("glomangiomas") localizes to chromosome 1p21-22. Am J Hum Genet 1999;65:125-33.

19 Neilsen DE, Brunger JW, Heeger S, Bamshad M, Robin NH. Mixed clefting type in Rapp-Hodgkin syndrome. Am J Med Genet 2002;108:281-4.

20 Frazer FC. Thoughts on the etiology of clefts of the palate and lip. Acto Genetica 1955;5:358-69.

21 Mamane Y, Heylbroeck C, Genin P, Algarte M, Servant MJ, LePage C, Deluca $C$, Kwon $H$, Lin R, Hiscott J. Interferon regulatory factors: the next generation. Gene 1999:3:B:1-14.

22 Au WC, Yeow WS, Pitha PM. Analysis of functional domains of interferon regulatory factor 7 and its association with IRF-3. Virology 2001;15:B, 273-82.

23 Escalante CR, Yie J, Thanos D, Aggarwal AK. Structure of IRF1 with bound DNA reveals determinants of interferon regulation. Nature 1998;391:103-6.

24 Vikkula M, Olsen BR. Unraveling the molecular genetics of osteoarthritis. Ann Med 1996;28:301-4

25 Vikkula M, Metsäranta M, Ala-Kokko L. Type II collagen mutations in rare and common cartilage diseases. Ann Med 1994;26:107-14.

26 Vikkula M, Mariman ECM, Lui VCH, Zhidkova NI, Tiller GE, Goldring M, van Beersum SEC, de Waal Malefijt MC, van den Hoogen FHG, Ropers $\mathrm{H}-\mathrm{H}$, Mayne R, Cheah KSE, Olsen BR, Warman ML, Brunner HG. Autosomal dominant and recessive osteochondrodysplasias associated with the COL11A2 locus. Cell 1995;80:431-7. 